\title{
Dynamic Correlation in Wave Propagation in Random Media
}

\author{
A.A. Chabanov, ${ }^{1,2}$ B. $\mathrm{Hu},{ }^{1}$ and A.Z. Genack ${ }^{1}$ \\ ${ }^{1}$ Department of Physics, Queens College of the City University of New York, Flushing, NY 11367, USA \\ ${ }^{2}$ CEMS, University of Minnesota, Minneapolis, MN 55455, USA
}

(Dated: February 20, 2004)

\begin{abstract}
We report time-resolved measurements of the statistics of pulsed transmission through quasi-onedimensional dielectric media with static disorder. The normalized intensity correlation function with displacement and polarization rotation for an incident pulse of linewidth $\sigma$ at delay time $t$ is a function only of the field correlation function, which is identical to that found for steadystate excitation, and of $\kappa_{\sigma}(t)$, the residual degree of intensity correlation at points at which the field correlation function vanishes. The dynamic probability distribution of normalized intensity depends only upon $\kappa_{\sigma}(t)$. Steady-state statistics are recovered in the limit $\sigma \rightarrow 0$, in which $\kappa_{\sigma=0}$ is the steady-state degree of correlation.
\end{abstract}

PACS numbers: 42.25.Dd, 42.25.Bs, 05.40.-a

The statistics of steady-state classical wave propagation [1, 2, 3] and electronic conductance [4] in disordered systems reflect the superposition of partial waves following trajectories with a wide distribution of path lengths, which is proportional to the particle time-of-flight distribution. Since the lengths of trajectories increase with time, paths cross upon themselves more frequently and the impact of weak localization could be expected to build in time [5, 6, 7, 8, 9, 10, 11, 12, 13, 14, 15]. This was demonstrated recently in the observation of an increase of the coherent backscattering enhancement from 2 to 3 in time-resolved acoustic measurements in a threedimensional elastic body [9], and in a time-decaying leakage rate of microwave radiation from a quasi-1D random dielectric sample [12]. To achieve a systematic understanding of weak localization in the time domain, it is essential to examine the statistics of propagation in addition to ensemble-averaged transport. This can be accomplished by parsing transmission according to the delay from an exciting pulse and studying the correlation and probability distribution of intensity as a function of delay time, and finally by relating these dynamics to steady-state statistics obtained under monochromatic excitation. A dynamical perspective on the impact of gain or loss on steady-state statistics might be of particular interest since the distribution of trajectories within the sample at a given delay is not altered by the presence of inelastic processes, while the average temporal profile changes.

Nonlocal intensity correlation 16, 17, 18, 19 leads to giant transmission fluctuations 3, 16, 17, 18, 19, 20, 21, 22, 23] and lies at the heart of mesoscopic physics 24]. The intensity correlation function associated with bulk scattering versus displacement or polarization rotation of a detector may be expressed as $C=F+\kappa(F+1)$ [18, 19, 23], where $F=\left|F_{E}\right|^{2}$ is the square of the field correlation function. The degree of correlation, $\kappa$, is the value of $C$ when $F$ vanishes, which occurs, for example, for a polarization shift of $90^{0}$ or for displacements much greater than a wavelength. In the absence of inelastic processes, the probability distributions of both intensity and total transmission normalized to their respective ensemble averages, $P\left(s_{a b}\right)$ and $P\left(s_{a}\right)$, where $a$ and $b$ are modes of the incident and transmitted waves, are obtained from diagrammatic 20] and random matrix 21 theories in terms of the dimensionless conductance, $g$ [25], in the diffusive limit, $g \gg 1$. For both quantum and classical waves, $g$ is equal to the sum of transmission coefficients over all input and output modes [26]. Surprisingly, theoretical expressions for $P\left(s_{a b}\right)$ and $P\left(s_{a}\right)$ 20, 21] closely match the measured distributions 22. even in the presence of absorption, and even at the localization threshold, reached when $g \sim 1$, when $g$ is replaced by $2 / 3 \operatorname{var}\left(s_{a}\right)$.

In this Letter, we report microwave measurements of the time-resolved field transmitted through random quasi-1D dielectric samples. Remarkably, the field correlation functions with displacement and polarization rotation at any time are identical to those found in steady state. We also find that the corresponding cumulant correlation functions of the normalized intensity have the same dependence upon the field correlation functions as in the frequency domain, $C_{\sigma}(t)=F+\kappa_{\sigma}(t)(F+1)$, with a parameter, $\kappa_{\sigma}(t)$, expressing the degree of correlation at time $t$ following excitation by a Gaussian pulse of width $\sigma$. We find further that the probability distribution of normalized intensity, $P\left(s_{a b}(t)\right)$, has the same form as for the steady-state transmitted intensity distribution [20, 21], but with $2 / 3 \kappa_{\sigma}(t)$ substituted for $g$. We find that even in diffusive samples, $\kappa_{\sigma}(t)$ reaches values exceeding the steady-state value at the Anderson localization threshold [27] of $\kappa \simeq 2 / 3$ [28]. Steady-state statistics are found to be a limiting case of dynamic statistics, in which the incident pulse linewidth vanishes, $\sigma \rightarrow 0$, with $\kappa_{\sigma=0}=\kappa$. These results show that $\kappa_{\sigma}(t)$ is the essential function describing the statistics of wave propagation.

Spectral measurements of the field transmission coefficient of microwave radiation as a function of rotation of 
linear polarization and displacement are made with use of a vector network analyzer in ensembles of random dielectric samples in which the wave is diffusive. The samples are contained in a copper tube with open ends of length $L$ greatly exceeding its 7.3 -cm diameter. New sample realizations are produced by briefly rotating the sample tube about its axis after each spectrum is taken. The response to a pulse with a Gaussian temporal envelope at carrier frequency $\nu_{0}$ is obtained by Fourier transforming the product of the field transmission spectrum and a Gaussian spectral function of width $\sigma$.

Polarization-selective measurements of the transmitted microwave field are made with use of a conical horn detector [19]. The samples are composed of 0.95-cm-diameter alumina spheres with refractive index 3.14 embedded in Styrofoam spheres of diameter $1.9 \mathrm{~cm}$ and refractive index 1.04 at an alumina volume fraction of 0.068. Measurements are made in an ensemble of 10,000 alumina configurations of $L=61 \mathrm{~cm}$ (Sample A) over the frequency range $14.7-15.7 \mathrm{GHz}$ in steps of $0.6 \mathrm{MHz}$ for a single orientation of the horn detector. Measurements are also carried out in an ensemble of 12,000 alumina configurations of $L=90 \mathrm{~cm}$ (Sample B) over the frequency range 16.95-17.05 GHz in 1-MHz steps, for 7 orientations of the horn detector rotated in steps of $15^{0}$ over a $90^{\circ}$ range. Steady-state measurements of intensity correlation give $\kappa=0.09$ in Sample A [12] and $\kappa=0.29$ in Sample B [19].

Examples of the response to excitation pulses with three different values of $\sigma$ in a single random realization of Sample A are shown in Fig. 1a versus time delay from the center of the incident pulses in units of the diffusion time, $t_{D}=\left(L+z_{0}\right)^{2} / \pi^{2} D$, where $D$ is the diffusion coefficient and $z_{0}$ is the boundary extrapolation length. The width of temporal intensity fluctuations is seen to be approximately equal to that of the incident pulse. This can be expressed quantitatively via the correlation function with time shift $\Delta t$ of the transmitted field normalized to the square root of the ensemble average of the time-varying intensity at time $t, F_{E_{\sigma}}=\left\langle E_{\sigma}^{*}(t) E_{\sigma}(t+\Delta t)\right\rangle / \sqrt{\left\langle I_{\sigma}(t)\right\rangle\left\langle I_{\sigma}(t+\Delta t)\right\rangle}$. We find that $F_{E_{\sigma}}$ is independent of $t$. In the case of a Gaussian incident pulse of bandwidth $\sigma,\left|F_{E_{\sigma}}\right|$ is a Gaussian with width $(\sqrt{2} \pi \sigma)^{-1}$ (Fig. 1b). We also find that, though $I_{\sigma}(t)$ in any given realization depends strongly upon $\sigma$, $\left\langle I_{\sigma}(t)\right\rangle$ for $t>t_{D}$ depends only weakly upon $\sigma$, once $\sigma>1 / \pi^{2} t_{D}$.

The correlation function of the normalized transmitted field with shift in polarization angle $\Delta \theta, F_{E}(\Delta \theta)$, is shown in Fig. 2a for the two delay times indicated by the vertical lines in the inset for the incident pulse of bandwidth $\sigma=5 \mathrm{MHz}$ and for monochromatic excitation. All functions are well described by the steady-state result, $F_{E}(\Delta \theta)=\cos (\Delta \theta)$ [19, 29]. The corresponding intensity correlation functions are shown in Fig. 2b. These have the form, $C_{\sigma}(\Delta \theta, t)=F(\Delta \theta)+\kappa_{\sigma}(t)[F(\Delta \theta)+1]$, with $\kappa_{\sigma}(t)=C_{\sigma}(90, t)$.
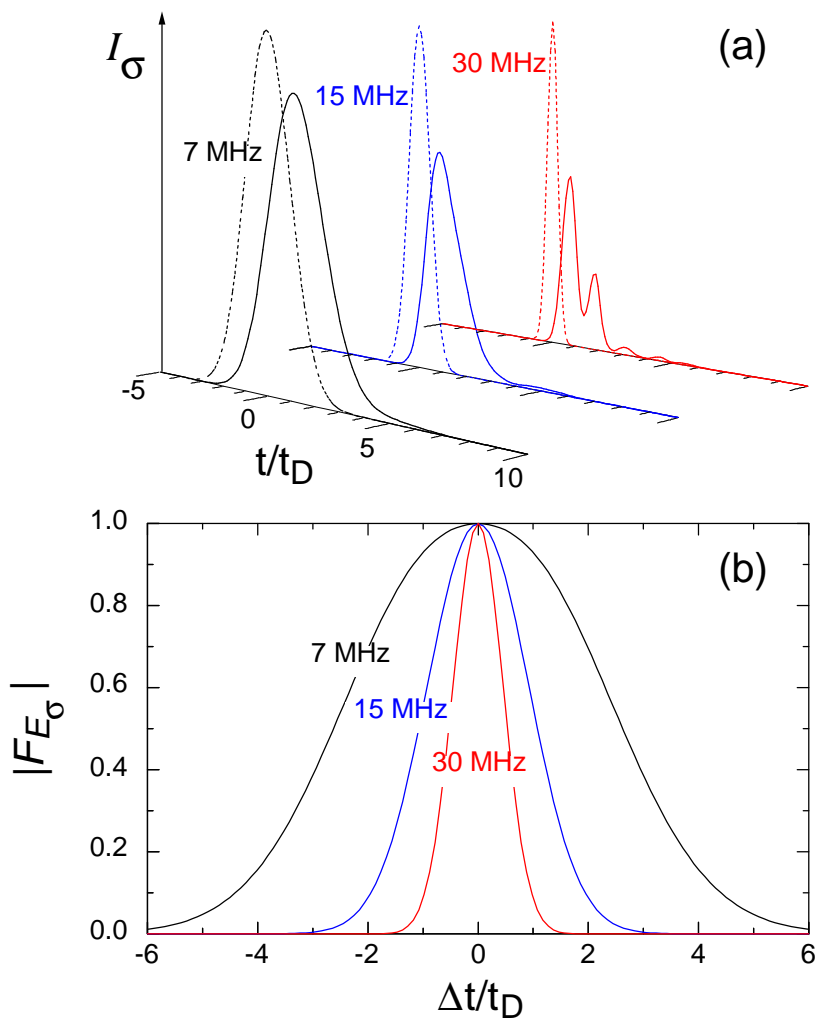

FIG. 1: (a) Transmitted intensity through a random realization of Sample A (solid curves) following incident Gaussian pulses (dashed curves) with $\sigma=7,15$, and $30 \mathrm{MHz}$. The incident pulses are centered at $t=0$ and shown with the same height. (b) Absolute value of the field correlation function of $E_{\sigma}(t) / \sqrt{\left\langle I_{\sigma}(t)\right\rangle}$ with time shift for the values of $\sigma$ in (a).

In order to study the dynamics of spatial correlation, measurements are taken at 50 points separated by 1.06 $\mathrm{mm}$ along a line centered on the tube axis on the output surface of a random sample of Polystyrene spheres [18]. A 3-mm antenna is aligned perpendicular to the line of displacement. The Polystyrene spheres of refractive index 1.59 are packed at a volume filling fraction of 0.52 in a copper tube with $L=100 \mathrm{~cm}$. Measurements are made in 1380 sample realizations over the frequency range 17.2$17.8 \mathrm{GHz}$ in steps of $0.625 \mathrm{MHz}$. In this sample, $\kappa=0.06$.

The correlation function of the normalized transmitted field with displacement $\Delta r, F_{E}(\Delta r)$, is shown in Fig. 3a for the two delay times indicated in the inset following pulsed excitation with $\sigma=20 \mathrm{MHz}$ and for monochromatic excitation. The overlap of these curves indicates that the dynamic $F_{E}(\Delta r)$ is independent of $t$ and identical with the steadystate field correlation function. The corresponding intensity correlation functions, shown in Fig. 3b, have the form, $C_{\sigma}(\Delta r, t)=F(\Delta r)+\kappa_{\sigma}(t)[F(\Delta r)+1]$, with $\kappa_{\sigma}(t)$ determined from the residual correlation at displacements $\Delta r>3.5 \mathrm{~cm}$, except for 

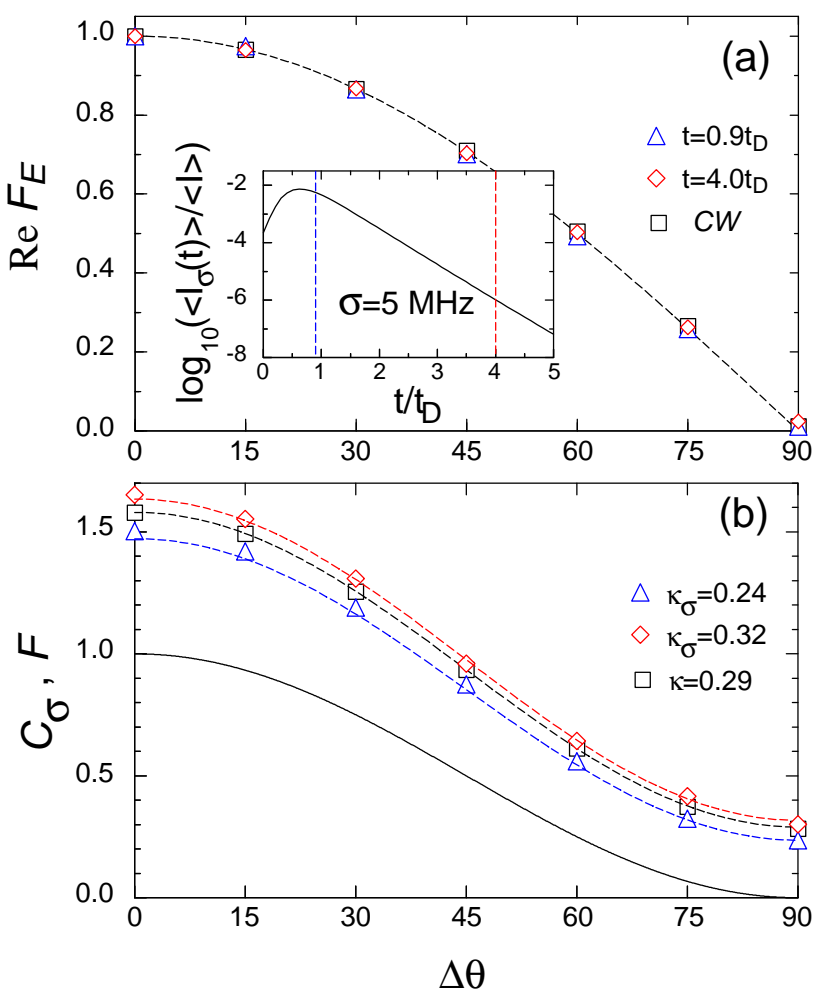

FIG. 2: (a) Real part of the field correlation function, $F_{E}(\Delta \theta)$, and (b) intensity correlation function, $C_{\sigma}(\Delta \theta)$, with polarization rotation of the transmitted wave through Sample B at the two delay times following pulsed excitation with $\sigma=5 \mathrm{MHz}$ and for monochromatic excitation $(C W)$. The dashed curves are (a) $F_{E}(\Delta \theta)=\cos (\Delta \theta)$ and (b) $C_{\sigma}(\Delta \theta, t)=F(\Delta \theta)+\kappa_{\sigma}(t)[F(\Delta \theta)+1]$ with the values of $\kappa_{\sigma}(t)$ indicated. The solid curve is $F(\Delta \theta)$. The logarithm of the average pulsed transmission through Sample B for $\sigma=5 \mathrm{MHz}$, normalized by the average steady-state transmitted intensity, is shown in the inset. The delay times at which correlation is presented in the figure are indicated by vertical dashed lines.

$t / t_{d}=4.8$ when $\kappa_{\sigma}(t)$ is determined from the relation, $\operatorname{var}\left(s_{a b}(t)\right)_{\sigma}=C_{\sigma}(0, t)=1+2 \kappa_{\sigma}(t)$.

The time-resolved probability distributions of normalized transmitted intensity, $P\left(s_{a b}(t)\right)$, are shown in Fig. 4 for the three samples considered, for various values of delay time and pulse bandwidth. The steady-state intensity distributions for Sample B and for the Polystyrene sample are also shown. Apart from the uppermost curve, each of the curves is displaced by a multiple of one decade for clarity of presentation. The dashed curves are obtained from the expressions for the steady-state intensity distribution $P\left(s_{a b}\right)$ 20, 21] in the limit, $g \gg 1$, and in the absence of absorption, but with $2 / 3 \kappa_{\sigma}$ substituted for $g$,

$$
P\left(s_{a b}\right)=\int_{0}^{\infty} \frac{d s_{a}}{s_{a}} P\left(s_{a}\right) \exp \left(-s_{a b} / s_{a}\right)
$$
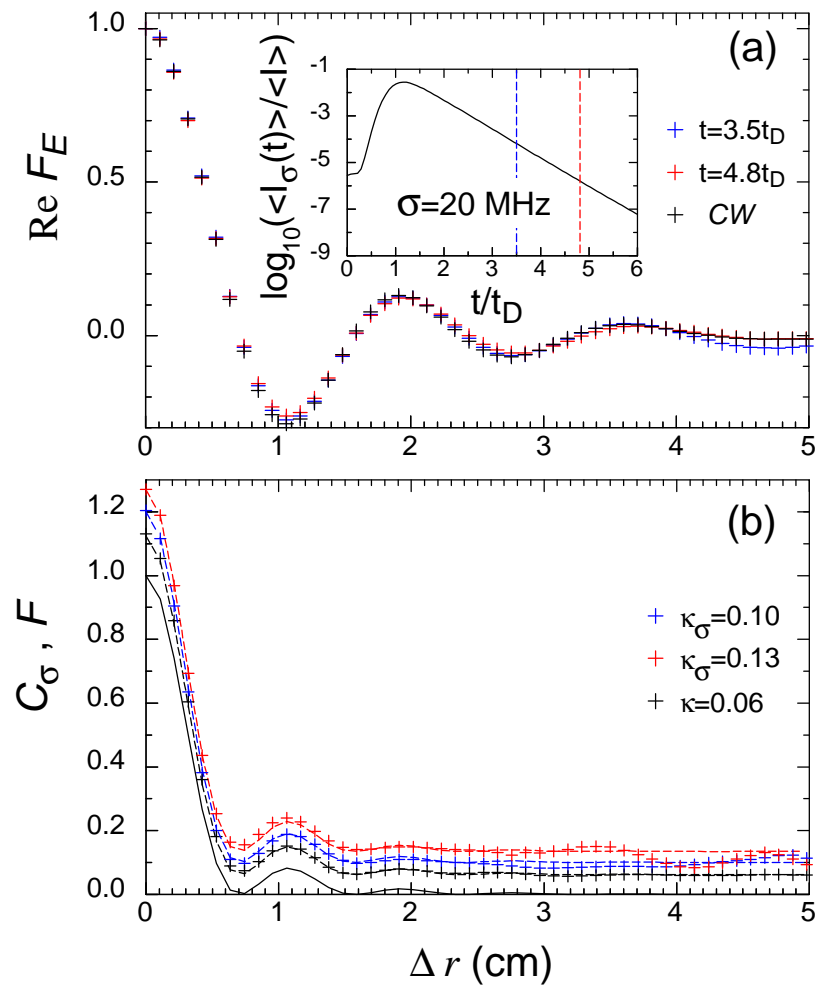

FIG. 3: (a) Real part of the field correlation function $F_{E}(\Delta r)$, and (b) intensity correlation function, $C_{\sigma}(\Delta r)$, with displacement on the output surface of a random Polystyrene sample at the two delay times following pulsed excitation with $\sigma=20 \mathrm{MHz}$ and for monochromatic excitation $(C W)$. The dashed curves in (b) are $C_{\sigma}(\Delta r, t)=F(\Delta r)+\kappa_{\sigma}(t)[F(\Delta r)+1]$, with the values of $\kappa_{\sigma}(t)$ indicated. The solid curve is $F(\Delta r)$. The logarithm of the average pulsed transmission through the sample for $\sigma=20 \mathrm{MHz}$, normalized by the average steady-state transmitted intensity, is shown in the inset. The delay times at which correlation is presented in the figure are indicated by vertical dashed lines.

with

$$
P\left(s_{a}\right)=\int_{-i \infty}^{i \infty} \frac{d v}{2 \pi i} \exp \left[v s_{a}-\Phi(v)\right]
$$

where

$$
\Phi(v)=\left(2 / 3 \kappa_{\sigma}\right) \ln ^{2}\left(\sqrt{1+3 v \kappa_{\sigma} / 2}+\sqrt{3 v \kappa_{\sigma} / 2}\right) .
$$

The values of $\kappa_{\sigma}$ for Sample B and for the Polystyrene sample are obtained from the measured intensity correlation functions in Figs. 2 and 3 . The values of $\kappa_{\sigma}$ for Sample A are determined from the relation, $C_{\sigma}(0, t)=1+2 \kappa_{\sigma}(t)$. Excellent agreement with measured results is obtained in all cases. These encompass both steady state and dynamic propagation, in the presence of strong and weak absorption, in the weak as well as the strong correlation regimes, in which $\kappa_{\sigma}$ exceeds its value at the Anderson localization threshold of 2/3. 


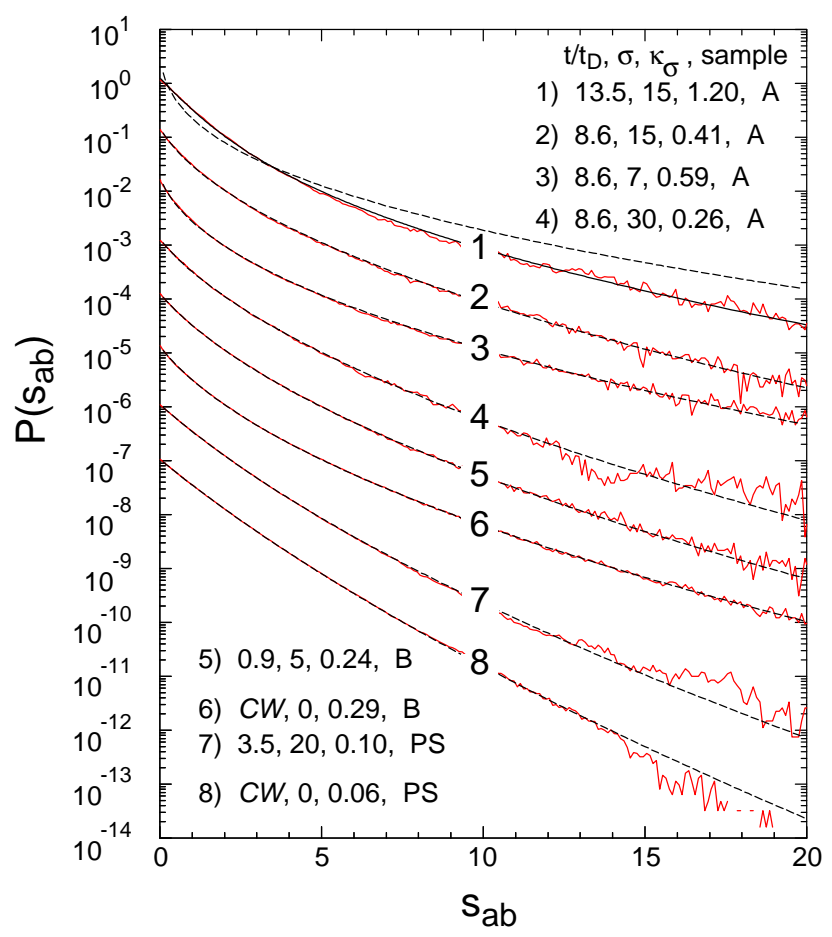

FIG. 4: Dynamic and steady-state $(C W)$ probability distributions of normalized transmitted intensity (solid curves) with the values of $t / t_{D}, \sigma$, and $\kappa_{\sigma}$, and sample type indicated. The dashed curves are from Eqs. (1-3). In the case of distribution (1) of long delay time, noise accounts for nearly half the measured field. The curve through the data is obtained by transforming accordingly Eqs. (1-3) to include the effect of noise. In the cases other than (1), noise is disregarded.

In conclusion, the time-resolved intensity correlation function has a universal form in terms of $\kappa_{\sigma}(t)$ and the square of the time-independent field correlation function, $F$. At the same time, the probability distribution of intensity is determined exclusively by $\kappa_{\sigma}(t)$. In the limit, $\sigma \rightarrow 0, \kappa_{\sigma} \rightarrow \kappa$, yielding steady-state statistics. Thus, the time-varying degree of correlation, $\kappa_{\sigma}(t)$, is the controlling function of mesoscopic statistics. It is, therefore, of prime importance to explore the possibility of a universal formulation of the time variation of the degree of correlation and its relationship to spatial localization.

We thank P. Sebbah for helpful suggestions. This research is sponsored by the National Science Foundation (DMR0205186) and U.S. Army Research Office (DAAD190010362).

[1] J.W. Goodman, Statistical Optics, (Wiley, New York, 1985).

[2] Scattering and Localization of Classical Waves in Random Media, edited by Ping Sheng (World Scientific, Singapore, 1990).
[3] M.C.W. van Rossum and Th.M. Nieuwenhuizen, Rev. Mod. Phys. 71, 313 (1999).

[4] Mesoscopic Phenomena in Solids, edited by B.L. Altshuler, P.A. Lee, and R.A. Webb (North Holland, Amsterdam, 1991).

[5] B.L. Altshuler, V.E. Kravtsov, and I.V. Lerner, Sov. Phys. JETP 67, 795 (1988); also in Ref. 4].

[6] B.A. Muzykantskii and D.E. Khmelnitskii, Phys. Rev. B 51, 5480 (1995); ArXiv: cond/mat9601045.

[7] A.D. Mirlin, Phys. Rep. 326, 259 (2000).

[8] V.N. Prigodin, B.L. Altshuler, K.B. Efetov, and S. Iida, Phys. Rev. Lett. 72, 546 (1994);

[9] R. Weaver and J. Burkhardt, J. Acoust. Soc. Am. 96, 3186 (1994); R.L. Weaver and O.I. Lobkis, Phys. Rev. Lett. 84, 4942 (2000).

[10] M. Titov and C.W.J. Beenakker, Phys. Rev. Lett. 85, 3388 (2000).

[11] H. Schomerus, K.J.H. van Bemmel, and C.W.J. Beenakker, Phys. Rev. E 63, 026605 (2001).

[12] A.A. Chabanov, Z.Q. Zhang, and A.Z. Genack, Phys. Rev. Lett. 90, 203903 (2003).

[13] M. Haney and R. Snieder, Phys. Rev. Lett. 91, 093902 (2003).

[14] S.E. Skipetrov and B.A. van Tiggelen, ArXiv: cond/mat0309381.

[15] S.K. Cheung, X. Zhang, Z.Q. Zhang, A.A. Chabanov, and A.Z. Genack, ArXiv: cond/mat0311184.

[16] M.J. Stephen and G. Cwilich, Phys. Rev. Lett. 59, 285 (1987); S. Feng, C. Kane, P.A. Lee, and A.D. Stone, Phys. Rev. Lett. 61, 834 (1988).

[17] N. Garcia and A.Z. Genack, Phys. Rev. Lett. 63, 1678 (1989); A.Z. Genack, N. Garcia, and W. Polkosnik, Phys. Rev. Lett. 65, 2129 (1990); J.F. de Boer, M.P. van Albada, and A. Lagendijk, Phys. Rev. B 45, 658 (1992).

[18] P. Sebbah, B. Hu, A.Z. Genack, R. Pnini, and B. Shapiro, Phys. Rev. Lett. 88, 123901 (2002).

[19] A.A. Chabanov, N.P. Trégourès, B.A. van Tiggelen, and A.Z. Genack, ArXiv: cond/mat0311184.

[20] Th.M. Nieuwenhuizen and M.C.W. van Rossum, Phys. Rev. Lett. 74, 2674 (1995).

[21] E. Kogan and M. Kaveh, Phys. Rev. B 52, R3813 (1995).

[22] M. Stoytchev and A.Z. Genack, Phys. Rev. Lett. 79, 309 (1997); M. Stoytchev and A.Z. Genack, Opt. Lett. 24, 262 (1999).

[23] S.-H. Chang, A. Taflove, A. Yamilov, A. Burin, and H. Cao, Opt. Lett. (2004) (to appear).

[24] R.A. Webb, S. Washburn, C.P. Umbach, and R.B. Laibowitz, Phys. Rev. Lett. 54, 2696 (1985); B.L. Altshuler and D.E. Khmelnitskii, JETP Lett. 42, 359 (1985); P.A. Lee and A.D. Stone, Phys. Rev. Lett. 55, 1622 (1985).

[25] D.J. Thouless, Phys. Rev. Lett. 39, 1167 (1977); E. Abrahams, P.W. Anderson, D.C. Licciardello, and T.V. Ramakrishnan, Phys. Rev. Lett. 42, 673 (1979).

[26] R. Landauer, Philos. Mag. 21, 863 (1970); E.N. Economou and C.M. Soukoulis, Phys. Rev. Lett. 46, 618 (1981); D.S. Fisher and P.A. Lee, Phys. Rev. B 23, 6851 (1981).

[27] P.W. Anderson, Phys. Rev. 109, 1492 (1958).

[28] A.A. Chabanov, M. Stoytchev, and A.Z. Genack, Nature, 404, 850 (2000).

[29] I. Freund, M. Kaveh, R. Berkovits, and M. Rosenbluh, Phys. Rev. B 42, 2613 (1990). 